\title{
A Search for Extraplanar Dust in Nearby Edge-On Spirals ${ }^{1}$
}

\author{
J. Christopher Howk \& Blair D. Savage \\ Department of Astronomy, University of Wisconsin-Madison, Madison, Wi. 53706 \\ Electronic mail: howk@astro.wisc.edu, savage@astro.wisc.edu
}

To appear in The Astronomical Journal, May 1999.

\begin{abstract}
We present high resolution $\left(00^{\prime \prime} 6\right.$ to $\sim 1^{\prime \prime}$. 0 ) BV images of 12 edge-on spiral galaxies observed with the WIYN 3.5-m telescope. These images were obtained to search for extraplanar $(|z|>0.4$ kpc) absorbing dust structures similar to those previously found in NGC 891 (Howk \& Savage 1997). Many of these galaxies have been previously searched for diffuse ionized gas at high $z$. Our imaged galaxies include a sample of seven massive $L_{*}$-like spiral galaxies within $D \lesssim 25$ Mpc that have inclinations $i \gtrsim 87^{\circ}$ from the plane of the sky. We find that five of these seven systems show extraplanar dust, visible as highly-structured absorbing clouds against the background stellar light of the galaxies. These dust structures lie at heights $|z| \gtrsim 0.4 \mathrm{kpc}$, which should be above most of the thin disk molecular material in these galaxies. The more prominent structures are estimated to have associated gas masses $\gtrsim 10^{5} \mathrm{M}_{\odot}$; the implied potential energies are $\gtrsim 10^{52}$ ergs. All of the galaxies in our sample that show detectable Ho emission at large $z$ also show extraplanar dust structures. None of those galaxies for which extraplanar Ho searches were negative show evidence for extensive high-z dust. The existence of extraplanar dust is a common property of massive spiral galaxies. We discuss several mechanisms for shaping the observed dust features. We emphasize in this discussion the possibility that these dusty clouds represent the dense phase of a multiphase medium at high- $z$ in spiral galaxies. In a few cases interactions with close galaxy companions could be responsible for the high- $z$ dust, either through dynamical stripping or triggered star formation. We can rule out warps as the source of the observed high- $z$ dust. Flaring gas layers seem an unlikely source of the observed material but cannot be ruled out at this time, except for those features that clearly connect to energetic processes in the disk. The correlation between high- $z$ dust and extraplanar $\mathrm{H} \alpha$ emission may simply suggest that both trace the high- $z$ interstellar medium in its various forms (or phases), the existence of which may ultimately be driven by vigorous star formation in the underlying disk. The absorption produced by high- $z$ dust and associated gas in spiral galaxies must be accounted for when studying extraplanar emission from spiral galaxies over much of the electromagnetic spectrum.
\end{abstract}

Subject headings: dust,extinction - galaxies: ISM - galaxies: spiral - galaxies: structure -ISM: clouds

\footnotetext{
${ }^{1}$ Based on observations obtained at the WIYN Observatory, a joint facility of the University of Wisconsin-Madison, Indiana University, Yale University, and the National Optical Astronomy Observatories.
} 


\section{INTRODUCTION}

Dust grains play an important role in the physics of the interstellar medium (ISM) in the thin disks of galaxies, affecting the heating and cooling of the gas, the phase structure of the medium, the distribution of metals, and the transfer of radiation. While the processes that are thought to circulate matter between the thin disks and halos of spiral galaxies will operate on both gas and dust, the dust content of interstellar thick disks and halos is poorly understood. In our own Galaxy there is evidence from gas-phase abundances that individual H I clouds at relatively large distances from the plane contain dust (Sembach \& Savage 1996, and references therein). However, these results give us little information on the distribution of dust with distance $z$ from the Galactic midplane.

In an earlier paper we presented high-resolution optical images of the nearby galaxy NGC 891 taken with the WIYN 3.5-m telescope (Howk \& Savage 1997, hereafter Paper I). These images reveal extensive amounts of highly-structured dust seen in absorption against the background stellar light of the galaxy. These dust structures, observable to heights $0.4 \lesssim z \lesssim 2.0 \mathrm{kpc}$ from the midplane, are seen along the entire length of the galaxy covered by the images presented in Paper I. We tentatively associated this dust absorption with the thick disk of interstellar material observed in other wavebands. An overly simple analysis of the radiative transfer, coupled with gas-to-dust relationships appropriate for the disk of the Milky Way (Bohlin, Savage, \& Drake 1978), suggests these dust structures are relatively opaque $\left(A_{V} \approx 0.8\right.$ to 2.0) with correspondingly large gas column densities $\left(N_{\mathrm{H}} \sim 10^{21}\right.$ atoms $\left.\mathrm{cm}^{-2}\right)$ and masses $\left(M \sim 10^{5}\right.$ to $\left.10^{6} \mathrm{M}_{\odot}\right)$. Estimates for the potential energies of the observed dusty clouds relative to the midplane are in the range $\Omega \sim 10^{52}-10^{53} \mathrm{ergs}$, similar to the energy estimates derived for Galactic supershells (Heiles 1979). The implied total gas mass associated with the ensemble of dusty high- $z$ clouds is roughly similar to that estimated for the mass of the extraplanar diffuse ionized gas (DIG) in NGC $891\left(\sim 10^{8} \mathrm{M}_{\odot}\right.$; Dettmar 1990). While there are uncertainties in these order of magnitude estimates, the images presented in Paper I clearly show that a substantial amount of dust is present in the thick disk of NGC 891.

How common is this phenomenon of extraplanar dust? Is it a peculiar feature of one galaxy, or is there evidence in the local universe that thick disk dust is a common feature of spiral galaxies? In this paper we try to partially answer these questions. We have undertaken a small imaging survey of edge-on galaxies within $D \lesssim 25 \mathrm{Mpc}$ using the WIYN 3.5-m telescope. Our sample is limited to northern-hemisphere galaxies with inclinations $i \gtrsim 87^{\circ}$ from the plane of the sky, though after obtaining our images, some of these galaxies seemed less highly inclined. Table 1 gives a summary of the properties of our imaged galaxies. The properties listed in Table 1 are taken from NEDF, except for the distances which are from Tully (1988) 3 . For each of these galaxies (with one exception) we have obtained 900s B and V images. Not all of the imaged galaxies are appropriate for the present work given the aforementioned inclination problems. We have exluded such galaxies from our "final sample." We also limit this final sample to only massive $L_{*}$ galaxies to avoid problems with large changes in the gas-to-dust ratios. We will show in this paper that the presence of highly-structured dust at large heights $(z \gtrsim 400 \mathrm{pc})$ from the midplane is a relatively common phenomenon and seems to be correlated with the presence of extraplanar DIG.

In $\$ 2$ we give the details of our observations and reductions; $\$ 3$ discusses our image display, particularly our approach to producing unsharp masks to more clearly reveal dust structures. Our primary observational

\footnotetext{
${ }^{2}$ The NASA/IPAC Extragalactic Database (NED) is operated by the Jet Propulsion Laboratory, California Institute of Technology, under contract with the National Aeronautics and Space Administration.

${ }^{3}$ The distance for NGC 4634 is not from Tully (1988). We adopt a distance $d=19$ Mpc from Teerikorpi et al. (1992)
} 
results are given in $\S$. In $\S 4.2$ we briefly discuss important properties of each of our target galaxies and give reasons for exluding particular galaxies from our final sample. A discussion of the implications for the origins of high- $z$ dust and for other observations of edge-on spirals is given in \$, and we summarize our main conclusions in $\$ 6$.

\section{OBSERVATIONS AND REDUCTIONS}

All of the observations presented here were obtained with the WIYN 3.5-m telescope at Kitt Peak National Observatory. The WIYN imager in use at the time of our observations is a thinned $2048 \times 2048$ STIS CCD with $21 \mu \mathrm{m}$ pixels. Placed at the f/6.5 Nasmyth focus of the WIYN telescope each pixel corresponds to $0{ }^{\prime \prime} 196$ on the sky. The imager has a $6 ! 7 \times 6 ! 7$ field of view. The images presented here were taken under non-photometric conditions, though the seeing conditions were generally good.

A log of our observations for each galaxy is given in Table 2. This table shows for each image the filter used, the date of observation, and the seeing-limited resolution for that exposure, expressed as the FWHM (in arcseconds) of Gaussian fits to the stellar images using the IRAFf routine IMEXAM. Also given is the linear resolution of our observations at the assumed distance of each galaxy (see Table 1). With one exception this survey is based upon 900s exposures of each galaxy in the Harris B and V filters available at WIYN. For the galaxy NGC 4013 we include only a 600s V-band exposure.

The images have been bias-subtracted and flat field-corrected in the usual manner within IRAF. The flat fields were derived from observations of the "Great White Spot" in the telescope dome. We do not have multiple exposures with which to correct for cosmic-ray contamination, with the exception of the V-band data for NGC 4565, but this does not seriously affect our results.

In principle subtle variations in the flat-field of the image could mimic dust absorption in our target galaxies. However, the vast majority of the dust features we observe cannot be referred to as "subtle." The imprint of small scale features in the CCD flat-fields is relatively constant within a given night, allowing us to search for features common to all galaxies that might be instrument related. Our experience suggests that the small-scale features present in the flat-field are usually not significant compared with the structures we are studying and are well removed in even single exposures. For our deeper imaging of a few galaxies (Howk \& Savage 1999a,b), we have taken exposures with the target galaxies shifted to different parts of the imager to avoid complications associated with flat-fielding errors. Comparing the final images with the individual frames, we are able to assess the probability of confusing flat-field structures with dust structures within these galaxies; we have found no real evidence for confusion of flat-field features with absorbing dust structures.

\footnotetext{
${ }^{4}$ IRAF is distributed by the National Optical Astronomy Observatories, which is operated by the Association for Research in Astronomy, Inc., under cooperative agreement with the National Science Foundation.
} 


\section{IMAGE DISPLAY AND UNSHARP MASK PROCEDURE}

Figures 112 present the final results of our imaging work. For each galaxy we show the V-band image and an unsharp masked version of the V-band data. Since the main results of our survey depend on our ability to effectively display our images, it is worth discussing in detail our approach. We depend on unsharp masks of the V-band data to show the wealth of structures that are present in many of these galaxies. A good example of the advantages to unsharp masks is Figure 1, which shows our images of NGC 891. The V-band image (bottom panel) is displayed to show the distribution of light along the entire length of the galaxy. With this display the light from the bulge is saturated and shows little in the way of distinguishable dust features. However, the unsharp mask of the V-band data (top panel) allows us to show the absorbing structures along the entire length of the galaxy in one display. This display more accurately shows the complexity of the absorbing structures that thread through the bulge area.

The unsharp mask of an image was derived by dividing the original image by a version smoothed with a Gaussian kernel. The FWHM of the Gaussian used to produce the smoothed image was 15 pixels $\left(3{ }^{\prime \prime} 0\right)$ for galaxies closer than $10 \mathrm{Mpc}$ and 7.5 pixels (1".5) for galaxies at greater distances (see Table 1). With this approach we remove the large-scale gradients in the background light of the galaxy, allowing us to display all parts of the galaxy in a uniform manner. The procedure tends to accentuate structures on scales smaller than smoothing kernel ( $\Delta \lesssim 120-150$ pc for our galaxies). This can be both an advantage and disadvantage. Most of the structures we find in these galaxies have minor axis scales similar or smaller to this, though not all (see $\$ 4.3$ ). In some sense this offsets the natural tendency to have one's eye drawn towards very large structures. Although we tend to identify structures only in computer displays of the science images (i.e., not the unsharp masks), the reader's eye may be drawn to the more small-scale structure in our unsharp masks.

Bright stars near a target galaxy can produce large artifacts in unsharp masks of images, destroying information over a disproportionately large area. We have produced our unsharp masks by dividing the original data by a smoothed image with such problem stars replaced by a two dimensional fit to the surrounding background light before the smoothing process. In a few cases we have also removed bright cosmic rays in the same manner. The replaced area is usually chosen to be a circular aperture of several times the FWHM of the seeing disk. For fainter examples, the aperture radius was typically twice the size of the seeing disk, while the brighter stars required apertures often in excess of five times the FWHM of the seeing disk. We have also removed the effects of CCD blooming from a few bright stars (e.g., Figures 1 and 6 for NGC 891 and NGC 4217, respectively). This extra processing step to reduce the effects of bright stars has been limited to stars lying on or near the target galaxies. In a few cases foreground stars lay in front of the bright light of a galaxy bulge; we have avoided removing stars in cases where the background to be fit comes from rapidly varying bulge light.

By dividing the original image by the smoothed image with bright stars and other features removed, we are able to keep bright stars from influencing large regions of the target galaxy, while at the same time showing where these stars lay with respect to the target. The latter point is important, because artifacts can be present in our unsharp masked images very near the position of a bright star. One can see examples of the detrimental effects of even relatively faint sources in Figure 6. In this image the stars well away from the galaxy have not been removed in the masking process, and therefore the unsharp mask process

\footnotetext{
${ }^{5}$ We choose to display the V-band rather than B-band data because the higher signal to noise ratio of our V-band images more than makes up for the greater opacity expected from the dust features in the B-band.
} 
introduces the low-intensity halos surrounding many of the stars (and background galaxies). Even relatively faint stars in our image of NGC 4217 exhibit extended halos in the unsharp mask of our V-band image. However one can see in this image that the very bright star just north of the galaxy that was removed before the smoothing process does not show a large-scale halo. There are artifacts present near the edges of this star, but it does not overwhelm the galaxy as it does in unsharp masks produced without removing it from the image.

In general one should be wary of subtle features near point sources or even bright extended objects in our unsharp masks. A lesson to this effect is the image of NGC 4565 shown in Figure 9. This galaxy shows a relatively prominent bulge (more so than most of the galaxies in our sample). The unsharp mask of the V-band image shows a faint halo coincident with the relatively sharp edge of the bulge light seen in the V-band image. Faint, large-scale artifacts (white in our unsharp mask) can be seen where the bulge of the galaxy is brightest. In particular, an artifact is present just to the south of the dust lane where the bright center of the galaxy, whose emission changes on small length scales, dominates the light. Within the bulge area itself there are point sources (presumably foreground stars) that are hidden by the light of the bulge in our V-band data. These have not been masked out, and seem to exhibit slight artifacts. NGC 4565 is a good example of artifact production in our unsharp mask procedure, since the galaxy has very little high- $z$ dust. We have been careful to avoid producing artifacts. This is part of the reason we use different size smoothing boxes for galaxies depending on their distances: we have found that this scale of smoothing produces the most reliable results.

While there are possible problems associated with the interpretation of the unsharp masks displayed here, one can see that this approach is much more effective at showing the reader the true extent and complexity of the dust features we see at high- $z$ in many of these galaxies.

\section{RESULTS}

\subsection{Extraplanar Dust in the Survey}

It is immediately clear from Figures 112 that many of the galaxies in our sample show evidence for dust above the central dust lane. Only dust that is highly structured or significantly overdense relative to its surroundings is expected to be obvious in our images. Thus there could be a smoothly-distributed component of dust at high- $z$ in all of these galaxies. For our purposes, we are interested in dust structures at heights $z \gtrsim 400$ pc, i.e., well above the central absorbing dust lanes in these galaxies. For comparison the FWHM of the thin disk molecular gas is estimated to be $\sim 220 \mathrm{pc}$ in NGC 891 (Scoville et al. 1993), $\sim 150$ pc in NGC 4013 (García-Burillo, Combes, \& Neri 1999, but adopting our assumed distance of $17 \mathrm{Mpc}$ ), and $\sim 100 \mathrm{pc}$ for the Milky Way (e.g., Scoville \& Sanders 1987). Thus structures with heights $z \gtrsim 400$ pc should lie above most of the material associated with the thin interstellar disks of these galaxies. In Paper I it was pointed out that the optical identification of dust features such as those seen in our images is susceptible to a number of selection effects. We are much more sensitive to absorbing structures that lie preferentially on the near side of a galaxy. In Paper I we characterized dust structures at high- $z$ in NGC 891 by their "apparent extinction," $a_{\lambda}$. We defined the apparent extinction in a waveband $\lambda$ as

$$
a_{\lambda}=-2.5 \log \left(S_{d c, \lambda} / S_{b g, \lambda}\right),
$$

where $S_{d c, \lambda}$ is the surface brightness measured towards a dust cloud, and $S_{b g, \lambda}$ is the surface brightness of the local background. The light measured towards a given dust feature contains an extincted component of 
starlight originating behind the feature along the line of sight as well as an unextincted component emitted in front of the feature. The apparent extinction in the V-band, $a_{V}$, is a lower limit to the true extinction, $A_{V}$. An absorbing structure that has a very large $A_{V}$ with a large amount of unextincted stellar light lying between it and the observer can have a very small $a_{V}$ and show too little contrast to be detectable. We cannot identify high- $z$ dust in galaxies with very thin stellar light distributions since we depend upon the background light of the stellar distribution to illuminate the dust in which we are interested.

Table 3 summarizes our observational results, listing our observational sample in order of decreasing star formation rate (SFR) per unit area of the visible disk. This quantity is traced by the far-infrared (FIR) luminosity, $L_{F I R}$, divided by $D_{25}^{2}$, the square of the major axis diameter defined by a surface brightness contour of $25 \mathrm{mag}$. $\operatorname{arcsec}^{-2}$ (e.g., Rand 1996). The FIR luminosities are derived from the IRAS $60 \mu \mathrm{m}$ and $100 \mu \mathrm{m}$ fluxes (Fullmer \& Lonsdale 1989). This tracer of the SFR of a galaxy is imperfect, however, given the contribution of the general interstellar radiation field to the heating of dust grains (see Rand 1996 for a discussion of this point). In particular the fraction of the FIR luminosity that is a result of heating by the interstellar radiation field may be a function of Hubble type (see Roberts \& Haynes 1994, and references therein).

Those galaxies that we have deemed appropriate to be included in our "final sample," i.e., massive $L_{*}$-like galaxies that are highly-enough inclined, are identified in Table 3. In practice we do not use the observed visual luminosity of our target galaxies to determine whether or not they should be included in this final sample of luminous spirals given the possibly very important effects of extinction in these edge-on systems. Instead we choose to use the observed H I velocity profiles of our targets as an indicator of their mass, and by extension their luminosity. Table 1 lists the velocity full-width at $20 \%$ peak, $W_{20}$, derived from the integrated H I profile for each of our target galaxies. These data are taken from the Third Reference Catalogue of Bright Galaxies (or RC3; de Vaucouleurs et al. 1991). We exclude galaxies from our sample if their rotation velocities (e.g., $W_{20} / 2$ ) are less than $200 \mathrm{~km} \mathrm{~s}^{-1}$ at the $3 \sigma$ level. Where possible we have used the detailed rotation curves of galaxies rather than the integrated $\mathrm{H}$ I profiles of the galaxies from the RC3 in making this judgement. We comment in $\$ 4.2$ on the reason(s) for excluding an imaged galaxy from this final sample, giving references for the detailed rotation curves where appropriate.

Various well-studied edge-on galaxies have not made it into the current survey for one reason or another. Well-known galaxies have often been excluded because they are too distant (e.g., NGC 5746, NGC 5775, and UGC 10288), have inclinations $i<87^{\circ}$ (e.g., NGC 3079, NGC 3556, and NGC 5023), are not spiral galaxies (e.g., NGC 4762), or have rotation velocities suggesting they are not massive $L_{*}$-like galaxies (e.g., NGC 3432, NGC 4244, IC 2233, and M 82). Our selection by inclination is quite subjective in most cases. For galaxies that were close to the cut-off we have usually excluded them from this sample on the basis of short WIYN images.

For each galaxy we make a judgement: is there evidence for significant extraplanar dust or not? Table 3 identifies those galaxies in our survey that show extraplanar dust with a $\bullet$ symbol, and those that show little or no evidence for high- $z$ dust with a $\bigcirc$ symbol. A few cases were ambiguous given their inclinations (e.g., NGC 4157 and NGC 4183). Also given in Table 3 is an indicator of the presence or absence of high- $z$ DIG, where a search has been made, and the general morphology of the DIG where present. References to the appropriate DIG studies are given in the table. An immediate conclusion that can be drawn from Table 3 3 is that every galaxy in our sample that was previously known to have high- $z$ DIG shows evidence for extraplanar dust and none of those lacking observable high- $z$ DIG shows any evidence for extraplanar dust.

A large fraction of the galaxies in our sample shows evidence for high- $z$ dust. Two cautionary comments 
are in order regarding our sample. The simpler of the two is that our sample size is quite small. We include seven galaxies in our final sample, five of which show extraplanar dust. The statistical uncertainties in a sample of this size are expected to be quite large. Assuming a binomial probability distribution we find a formal result of $(71 \pm 17) \%$ of spiral galaxies in the local universe have highly-structured extraplanar dust. However, our second caution is that our sample, in all likelihood, is neither statistically complete nor unbiased. We have subjectively judged the inclination of the galaxies which have poorly-known inclinations and chosen only those that visually appear to have inclinations $i \gtrsim 87^{\circ}$. We cannot guarantee we have included all appropriate galaxies, though most large galaxies in the local universe (outside of the zone of avoidance) are probably known. To our knowledge our final sample contains all of the truly edge-on $L_{*}$-like northern spirals within $25 \mathrm{Mpc}$. However, we are likely biased to large, well-studied galaxies, particularly those that have previously been searched for high- $z$ DIG. Another possibly important aspect of Table 3 is the non-uniformity of the observations of high- $z$ DIG. Even the individual galaxies within the larger surveys of Rand (1996) and Pildis, Bregman, \& Schombert (1994) have varying sensitivities to $\mathrm{H} \alpha$ emission.

Though the searches for high- $z$ DIG are not statistically rigorous, a picture has been developed based upon observations of more than 25 galaxies that couples the presence of DIG at high- $z$ with the SFR of the underlying disk. And though not many galaxies show bright, widespread diffuse $\mathrm{H} \alpha$ emission at high- $z$, like NGC 891, many of the galaxies studied (in the range of 60-70\%) show some form of high- $z$ DIG (e.g., Rand 1998; Dettmar 1998). However, the observations of this sample of galaxies are inhomogeneous. If we assume the presence of dust and ionized gas at high- $z$ in spiral galaxies is tied to the SFR, perhaps a more meaningful number might be the number of spiral galaxies with SFRs similar to those in our sample.

Galaxies with SFRs similar to NGC 4013 and NGC 891 are likely relatively common. For example, the Milky Way has a value of $L_{F I R} / D^{2} \sim 3 \times 10^{40} \mathrm{ergs} \mathrm{s}^{-1} \mathrm{kpc}^{-2}$ (Cox \& Mezger 1989; see Rand 1996), which is midway between the values of this quantity for NGC 891 and NGC 4013. The total $L_{F I R}$ of the Galaxy is estimated to be $\sim 4 \times 10^{43} \mathrm{ergs} \mathrm{s}^{-1}$, or $\sim 10^{10} L_{\odot}$ (Cox \& Mezger 1989). Roberts \& Haynes (1994) have compiled a volume-limited sample of galaxies in the Local Supercluster culled from the RC3 for comparing the properties of galaxies along the Hubble sequence. In their sample of Sab,Sb galaxies they derive a median FIR luminosity of $L_{F I R}=[3.7(1.3,7.9)] \times 10^{9} L_{\odot}$, where the numbers in parentheses give the the 25 th and 75 th percentile ranges. This median is based on a sample of 171 galaxies. Their sample of $214 \mathrm{Sbc}, \mathrm{Sc}$ galaxies gives a median $L_{F I R}=[4.8(1.5,11.5)] \times 10^{9} L_{\odot}$. The median value of $L_{F I R}$ for both our full and final samples is $L_{F I R}=3.7 \times 10^{9} L_{\odot}$ (using the upper limits as detections). These values are comparable to the FIR luminosity distribution in the Roberts \& Haynes sample, suggesting that our small sample of galaxies is not unusual compared with a larger sample in the Local Supercluster, at least as judged by the median values of $L_{F I R}$. Further, the FIR luminosities of the individual galaxies in our sample are not extraordinary given the broad distribution of $L_{F I R}$ in the Roberts \& Haynes sample. Though $L_{F I R}$ is an imperfect measure, this suggests the SFRs in our target galaxies may also typical of galaxies in the local universe.

The presence of dust at large distances from the midplane can no longer be viewed as a peculiar property of a single galaxy (e.g., NGC 891). Our observations reveal that the existence of inhomogeneously distributed (clumped) high- $z$ dust is a common property of $L_{*}$-like spiral galaxies. 


\subsection{Notes on Individual Galaxies}

We comment here briefly on the important properties of the individual galaxies in our sample. In particular we point out those galaxies that are not included in our final sample and describe why they were left out. We also comment on galaxies known to be interacting or in close groups, as well as those for which we have obtained more extensive WIYN imaging that will be presented in future works.

NGC 891 - This galaxy has long been known to harbor extraplanar dust (e.g., Sandage 1961; Keppel et al. 1991) and has been extensively studied at many wavelengths (see Paper I and references therein). We have obtained deeper BVI and $\mathrm{H} \alpha$ images for this galaxy and will present these results elsewhere (Howk \& Savage 1999a). NGC 891 is known to have an extensive distribution of DIG at high- $z$ (Dettmar 1990; Rand, Kulkarni, \& Hester 1990; Dettmar \& Schulz 1992; Rand 1996, 1997). There is no evidence for a warp and the evidence for an flare in the H I layer has recently been called into question (Sancisi \& Allen 1979; Swaters, Sancisi, \& van der Hulst 1997). Alton et al. (1998) have recently presented submillimeter $(\lambda 850 \mu \mathrm{m})$ images of NGC 891; they seem to detect emission from dust up to $z \sim 2 \mathrm{kpc}$ from the midplane, confirming our results of Paper I.

NGC 3628 - This well-studied galaxy is part of the Leo Triplet, which includes the galaxies NGC 3627 and NGC 3623. It is experiencing a close interaction with these nearby galaxies. The western edge of NGC 3628 shows a strong asymmetrical flare or warp seen in both H I and optical images (e.g., Wilding, Alexander, \& Green 1993). The observed extraplanar dust in this system may be the result of the strong interaction with its neighbors, either through gravitational stripping, extreme flaring, or induced star formation. However, Wilding et al. comment that within the central $4^{\prime}$ of the galaxy the H I disk shows very little high- $z$ emission and is close to unresolved at their $15^{\prime \prime}$ resolution. This galaxy houses a circumnuclear starburst and has an extended hot gaseous halo (Dahlem et al. 1996).

$N G C 4013$ - This galaxy shows extensive high- $z$ dust in our WIYN images. We have obtained deeper BVI and $\mathrm{H} \alpha$ images of this galaxy, the results of which will be presented elsewhere (Howk \& Savage 1999b). Rand (1996) has found faint diffuse $\mathrm{H} \alpha$ emission originating from high- $z$ DIG in this galaxy. García-Burillo et al. (1999) have recently reported on high-resolution $\left(\sim 3^{\prime \prime}\right)$ CO observations of this galaxy. Several of the high- $z$ filaments in their channel maps correspond to dust structures seen in our images. While a detailed comparison of the $\mathrm{CO}$ emission and the structures seen in our images is beyond the scope of our paper, we note that a dust feature ${ }^{6}$ projected against the bulge of the galaxy, stretching to $z \sim 750 \mathrm{pc}$, is coincident with an extraplanar CO feature in the maps of García-Burillo et al. (1999). The velocity of this feature is $-104 \mathrm{~km} \mathrm{~s}^{-1}$ with respect to the systemic velocity of the galaxy. This feature is almost directly above the dynamical center of the galaxy; if it were participating in the circular rotation of the galaxy (as in a flare), the velocity relative to systemic should be nearly zero. We believe this argues against this particular feature being a result of a flared gas layer at large distances from the center of the galaxy. NGC 4013 is known to have a prodigious H I warp perpendicular to the line of sight (line of nodes parallel to the line of sight), and possibly also a flared H I layer (Bottema 1995, 1996). The onset of the H I warp coincides with the end of the optical disk (Bottema 1995). We do not believe warps can explain the high- $z$ dust structures we observe in our target galaxies; we will discuss this issue in $\$ 5.1$.

$N G C 4157$ - The inclination of this galaxy makes the identification of true high- $z$ dust ambiguous given the geometric projection effects. We do not include it in our final sample but present the images here for the reader's benefit.

\footnotetext{
${ }^{6}$ This particular feature is identified as "Cloud 4" in Howk \& Savage 1999c.
} 
$N G C 4183$ - This galaxy may suffer from inclination effects as for NGC 4157. Also the rotational velocity of this galaxy suggests that it may not be $L_{*}$-like in mass (see Rhee \& van Albada 1996). We do not include this galaxy in our final sample.

$N G C 4217$ - This galaxy shows extensive high- $z$ dust along most of its length, including one spectacular loop to the SW of the galaxy center that can be traced to $z \sim 1.5 \mathrm{kpc}$ (NGC 4217:D $-016+015$ in Table 1 and Figure 14). We have obtained deeper BVI and $\mathrm{H} \alpha$ images of this galaxy, the results of which will be presented elsewhere (Howk \& Savage 1999b). Rand (1996) has identified two faint patches of $\mathrm{H} \alpha$ emission originating from high- $z$ DIG in this galaxy. The estimated SFR of this galaxy, derived from its $L_{\mathrm{FIR}}$, is quite low. Thus it does not fit the rough correlation observed between the presence of high- $z$ DIG (as well as dust) and SFR (see Rand 1996 and Dettmar 1998).

$N G C 4302$ - Large scale loops and filaments of dust are seen at high- $z$ along most of the length of this galaxy. It forms a close pair on the sky with NGC 4298. NGC 4302 itself shows little evidence for interaction, although NGC 4298 appears slightly distorted in the direction of NGC 4302. The DIG of this galaxy has been studied by Rand (1996) who finds a relatively widespread, diffuse layer of $\mathrm{H} \alpha$ emission detectable to $z \approx 2 \mathrm{kpc}$. The RC3 gives $W_{20}=377 \pm 8$ for this galaxy. Thus while its measured rotation velocity is less than our cut-off of $W_{20} / 2>200 \mathrm{~km} \mathrm{~s}^{-1}$, it is consistent with this value at the $3 \sigma$ level. Following Roberts \& Haynes (1994) we derive a total mass for this galaxy of $\sim 9.6 \times 10^{10} \mathrm{M}_{\odot}$, which is quite close to the other massive galaxies included in our final sample, most of which weigh in with $\sim 1-3 \times 10^{11}$ $\mathrm{M}_{\odot}$. We therefore include NGC 4302 in our final sample.

$N G C 4517$ - This galaxy appears to be similar to NGC 4565 in its inclination and high- $z$ dust content. However, it has a less prominent bulge, which makes the identification of high- $z$ dust more difficult. We find a few structures that may be dust at large $z$, but like NGC 4565 we find little evidence for widespread thick disk dust like that seen in NGC 891. Given the ambiguity caused by the inclination of this galaxy, and the lack of light against which to view dust absorption, we exclude this galaxy from our final sample.

$N G C 4565$ - This galaxy shows very little evidence for widespread extraplanar dust like that seen in NGC 891 and several other galaxies in our sample. However, there are two rather prominent extensions of dust seen to the NW of the bulge in Figure 9. One of these features is identified in Table 1 and Figure 15 (see $\$ 4.3$ ) as NGC 4565:D -064-016. Given the lack of similarity between this galaxy and the others showing high- $z$ dust, we classify it as a galaxy without significant amounts of high- $z$ dust in Table 3 . NGC 4565 shows evidence for both an optical (Naeslund \& Joersaeter 1997) and H I warp (Rupen 1991). Though there is no evidence for $\mathrm{H} \alpha$ or radio continuum emitting halos (Rand, Kulkarni, \& Hester 1992; Sukumar \& Allen 1991; Dumke et al. 1995), this galaxy does show high- $z$ X-ray emission (Vogler, Pietsch, \& Kahabka 1996). Given the absence of high- $z$ dust, this galaxy provides useful information about artifacts introduced by the unsharp masking process.

$N G C 4631$ - This galaxy is experiencing a close interaction with the nearby galaxies NGC 4656 and NGC 4627. The observed extraplanar dust in this system may be caused by a mechanism different than that responsible for high- $z$ dust in the more isolated galaxies of our sample, such as tidal stripping or other dynamical effects. Observations of H I emission in this system (Weliachew, Sancisi, \& Guélin 1978) show large amounts of gas between NGC 4631 and NGC 4656, presumably stripped from one or both; these observations also indicate that the H I disk of NGC 4631 is warped or disturbed. This galaxy is not included in our final sample because it rotates more slowly than one expects for a massive $L_{*}$-like galaxy (see Weliachew et al. 1978). High- $z$ DIG in this galaxy has been imaged by Rand, Kulkarni, \& Hester (1992) and studied spectroscopically by Golla, Dettmar, \& Domgörgen (1996). NGC 4631 is also known 
to have a very extended radio continuum halo with vertically oriented magnetic fields (Dumke et al. 1995; Hummel, Beck, \& Dahlem 1991).

$N G C 4634$ - We find this galaxy has an extended thick disk or halo of emitting material (presumably stars) that seems to flare with increasing projected distance from the center. Near the center of the galaxy, this extended light distribution seems to have a projected half-thickness of $\gtrsim 2 \mathrm{kpc}$, assuming a distance of 19 Mpc (Teerikorpi et al. 1992). Dettmar (1998) has presented an $\mathrm{H} \alpha$ image of this galaxy showing a bright DIG layer. This galaxy is not included in our final sample because of its low rotational velocity.

NGC 5907 - This well-studied Sc galaxy is characterized by a relatively low SFR and has no detectable high- $z$ dust or ionized gas (Rand 1996). NGC 5907 shows a warp in the optical (Morrison et al. 1994; Sánchez-Saavedra, Battaner, \& Florido 1990) and in H I emission (Sancisi 1976; Shang et al. 1998).

\subsection{Properties of Individual Dust Features}

In Figures 13 through 15 we show close-up views of some of the more prominent individual dust structures in our target galaxies. The properties of each feature shown in Figures 1315 are summarized in Table 5 . This table lists the ID of each feature as well as its position in J2000 equatorial coordinates. These positions have been determined with respect to stars common to our images and the Digitized Sky Survey and should be accurate to $\sim 1^{\prime \prime}$. Throughout this section we will use a common naming scheme to identify these individual features. We designate a structure using the form NGC GGGG:D $\pm \mathrm{XXX} \pm \mathrm{ZZZ}$. Here the NGC number of the individual galaxy is given as GGGG (clouds in UGC galaxies should have a parallel naming scheme UGC GGGG:D $\pm \mathrm{XXX} \pm \mathrm{ZZZ}$ ), and the "D" denotes a dust cloud. The value XXX is the projected distance in arcsec of the dust feature from the optical center of the galaxy traced along the major axis. Positive values are east of the center. The value ZZZ is the projected distance in arcsec of the dust feature from the midplane of the galaxy traced along the minor axis. Positive values are north of the midplane. Applying this naming scheme to feature 2 from Paper I $\left[(\alpha, \delta)_{\mathrm{B} 1950}=\left(2^{h} 19^{m} 20.4^{s}, 42^{\circ} 06^{\prime}\right.\right.$ $\left.44^{\prime \prime}\right)$ ] we have NGC 0891:D $-044+032$. The position of the midplane has roughly been determined for this purpose using the distribution of galaxy light as a function of height above the plane. We have determined the point of symmetry in the absorption of the central dust lane in cuts perpendicular to the galaxy plane in each of our images, and we adopt this reference point in specifying ZZZ. The center of the galaxy in the radial direction is similarly determined using cuts parallel to the major axis. These designations are only meant to be approximate to give the reader a sense of their radial and vertical positions in the galaxy, and the equatorial coordinates should be used for making detailed comparisons with other wavebands. In the case of NGC 4302, whose position angle is almost $0^{\circ}$, we define positive XXX values as towards the north, and positive ZZZ towards the west to maintain a consistent orientation of the coordinate system in the sense that the positive $z$-axis is clockwise from the $x$-axis in our figures.

In Table 4 we give approximate $z$-heights as well as rough dimensions of each structure. We also give the apparent extinctions $a_{B}$ and $a_{V}$ in the $\mathrm{B}$ and $\mathrm{V}$ bandpasses, respectively, as defined in Eqn. (何). We have measured these values as described in Paper I. The errors in these measurements are approximately $0.05-0.10$ mag. As we pointed out earlier, the apparent extinction measured towards an object is not equal to the true extinction because of foreground stellar light contamination and the effects of scattering of background stellar light into the structure. The apparent extinction does, however, represent a lower limit to the true extinction in a given bandpass. With information in three colors one can attempt to roughly disentangle the amount of true extinction (e.g., see Paper I); however, applying this rough 
approach to only two bandpasses seems dubious. In Table 1 we estimate a lower limit to the hydrogen column density of associated gas for each dust feature. We assume Galactic gas to dust relationships and $R_{V} \equiv A_{V} / E(B-V)=3.1$, which is the average for diffuse clouds in the Galactic disk (e.g., Cardelli, Clayton, \& Mathis 1989). Using the results of Bohlin et al. (1978) for the relationship of $N_{\mathrm{H}}$ with $E(B-V)$ with our assumed $R_{V}$, we estimate the column density as

$$
N_{\mathrm{H}}>1.9 \times 10^{21} a_{V}\left[\mathrm{~cm}^{-2}\right] .
$$

We also then give a lower limit to the mass of each feature derived from the product of this estimated column density and the projected area of the feature. The addition of light from stars residing in front of the dust features is not the only effect that tends to lower the apparent extinction from the true extinction. Another possibly significant effect is the unknown contribution of scattering off of dust grains and into the line of sight. This serves to lessen the perceived extinction and make it more "grey," i.e. less wavelength dependent (see Paper I). Indeed, the effects of the ill-constrained radiative transfer can be seen in Table 4 If $R_{V} \approx 3.1$ we expect $A_{B}=1.34 A_{V}$. If our dust feature lay between the observer and all of the starlight of the galaxy, and scattering were negligible, we would expect to also find $a_{B}=1.34 a_{V}$. The absorbing structure NGC 4013:D $+032-012$, for example, shows $a_{B} \approx 1.1 a_{V}$. This could be a result of the radiative transfer or of an incorrect $R_{V}$.

As in Paper I the structures we present here represent the more prominent ones and likely arise preferentially on the near side of each galaxy. The last column of Table fives a subjective description of the morphology of each structure. Note that the cloud boundaries outlined in Figures 13 and 14 are imprecise and very subjective. The identification of individual "clouds" in these galaxies is not only challenging, but in many cases perhaps inappropriate. The features we show may be part of larger structures, or they may simply be the result of overlapping structures along the line of sight. However, it was noted in Paper I that structures identified in WIYN images of NGC 891 generally did not break up in an image taken with the Hubble Space Telescope (HST). While the HST image presented in Paper I showed more substructure than was apparent in our WIYN images, it did not offer immediate evidence for a separation of our identified dust features into multiple structures.

Many of the structures have observed widths approximately twice our seeing-limited resolution. This suggests that the limited resolution of our images may be affecting our measurements. In these cases the true width may be lower than that quoted in Table 4 . In Paper I we compared the widths derived from our WIYN images and the archival HST image. The minor axis lengths measured in both the WIYN and HST images seemed to agree, however the results are somewhat dependent upon the subjective definition of the cloud boundaries. The linear resolution of the images presented in Paper I (and the NGC 891 images presented here) are significantly better than for most of our current dataset. For the more distant galaxies in our sample, the limited resolution of our images may be adversely affecting our measurements. This is true for our measurements of $a_{B}$ and $a_{V}$ as well, in the sense that observed at higher resolution the values of $a_{B}$ and $a_{V}$ may be greater.

The values $N_{H}$ of gas associated with the identified dust features shown in Table $\mathbb{A}$, which assume Galactic gas to dust relationships (see Paper I), are in the range $N_{\mathrm{H}}>3 \times 10^{20}-10^{21} \mathrm{~cm}^{-2}$. Of course our selection of features is biased towards the more opaque structures (and hence higher column density), but the true $A_{V}$ of a given dust feature may be significantly greater than the $a_{V}$ listed in Table 4 . The masses derived for these structures are similar to those derived for dust features in NGC 891 in Paper I. We include two dust absorbing structures from NGC 891 in our current sample so that the reader may compare these structures with those identified in our other galaxies. We have remeasured the properties of features 2 
and 7 from Paper I, or NGC 0891:D -044 + 032 and NGC 0891:D - 012 - 030, respectively, in the current nomenclature, and present them with the other structures in Table 1 and Figure 13. Our results suggest that the dust features observed in the more extended dataset presented here are similar to those discussed in Paper I for NGC 891. This statement is based more on impressions than a vigorous comparison, which would require a less biased approach to identifying such structures.

The potential energies of the identified structures are likely quite large. The structures identified in Table 1 in common with Paper I, NGC 0891:D - 044 + 032 and NGC 0891:D - 012 - 030, were estimated to have potential energies relative to the midplane $\Omega \sim 3 \times 10^{52}$ in that earlier work. Though the true potential energy depends sensitively on the variation of the gravitational acceleration with $z$ for a given galaxy (and hence on the mass scale height and midplane density), these features from NGC 891 are not unusual compared with the other features identified in Table 4 . This suggests that the potential energies of these other structures may also be $\Omega \gtrsim 10^{52} \mathrm{ergs}$, assuming the derived masses are appropriate.

One can see from Figures 13 15 that there is a large morphological diversity within the population of dust structures found at high- $z$ in a given galaxy. Evidently the high- $z$ ISM in these galaxies is non-uniform. We are able to observe these dusty clouds because they contain a greater density of dust (and perhaps gas) than their surroundings. There may very well be a more smoothly distributed component of dust in these galaxies. The observed range of morphologies need not imply a range of mechanisms for expelling material from the thin interstellar disks of these galaxies. The specific morphology of a given parcel of material will depend sensitively on the details of its current and past environments.

\section{DISCUSSION}

We have shown through images obtained with the WIYN observatory that dust can be found at large distances from the midplanes of many nearby spiral galaxies. In Paper I we discuss several possible mechanisms for shaping the dust seen far from the plane of NGC 891, all of which required the ejection of dusty material from the thin disk of that galaxy. Promising mechanisms include hydrodynamic or magnetohydrodynamic flows, such as fountain (Shapiro \& Field 1976; Bregman 1980; Houck \& Bregman 1990; de Avillez, Berry, \& Kahn 1998) and chimney (Norman \& Ikeuchi 1989; see also Heiles 1990) flows; expulsion via radiation pressure on dust grains (e.g., Davies et al. 1998; Ferrara 1993; Ferrara et al. 1991; Franco et al. 1991); and flows driven by magnetic field instabilities (e.g, Parker 1966, 1992). We refer the reader to Paper I for a detailed discussion of these models and how they might help to shape the observed high- $z$ dust structures.

We concluded in Paper I that star formation, and the resulting energy input into the ISM through radiation, stellar winds, and supernova explosions, must play a role in whatever process(es) one imagines is responsible for the dusty thick disk of material in NGC 891. That conclusion is strengthened in the current work by the striking correlation between the presence of high- $z$ dust and the detectability of

thick distributions of DIG in our sample of galaxies. Extraplanar DIG in galaxies has been studied more thoroughly than the dust structures we consider [cf., reviews by Dettmar (1998) and Rand (1998), and references therein]. The main finding of these works is a connection of the DIG emission with the SFR of the underlying disk. With very few exceptions, DIG far from the plane is found only in those disk galaxies showing large SFRs (traced again by the $L_{F I R}$ derived from IRAS observations). The correlation of high- $z$ DIG and dust found in the current work suggests that both phenomena must be ultimately tied to the same overall driving force, i.e., star formation. 


\subsection{The Effects of Gaseous Warps and Flares}

The observed dust structures cannot be due to gaseous warps in our target galaxies. We showed in Paper I that the rough number of dust features was similar for each side of NGC 891, which is not expected in the case of a warp along the line of sight; a warp in the plane of the sky is ruled out from H I observations (e.g., Swaters et al. 1997). Furthermore, the galaxies in our sample that do show prominent warps, NGC 3628, NGC 4013, NGC 4565, and NGC 5907, include two galaxies that show no evidence for substantial amounts of high- $z$ dust and two that show extensive high- $z$ dust (see Table 3). The influence of flared gas layers in our target galaxies is less easy to dismiss, i.e., the observed high- $z$ dust could be due to flares. However, we do not believe this is the case. The morphologies of some features are highly suggestive of hydrodynamical shaping by star formation (see Paper I), and the correlation presented here between high- $z$ dust and ionized gas also suggests a connection with the SFR of the disk. Many of the galaxies containing DIG at high- $z$ show either very patchy $\mathrm{H} \alpha$ emission, presumably associated with locally enhanced SFRs in the disk (e.g., above spiral arms), or thick disk DIG layers that cut off sharply at a given projected distance from the galaxy center (e.g., Rand 1996; Dettmar 1990; Rand et al. 1990). Neither of these observational phenomena is explained if the DIG at large projected distances from the midplane is due to a flared gas layer. We believe this argues against flares as an explanation for the thick DIG layers of galaxies, and by extension against flares as an explanation for high- $z$ dust. The aforementioned coincidence of dust features in NGC 4013 with extraplanar CO clouds (García-Burillo et al. 1999) at velocities incompatable with galactic rotation further suggests that at least some of these dusty clouds are not associated with a gaseous flare.

\subsection{A Multi-Phase ISM at High- $z$}

Though the high- $z$ ionized gas and dust observed in spirals seem to be highly correlated, we do not believe that they trace the same material. The clouds traced by dust, if they contain significant amounts of gas, must be denser than that traced by the general DIG. In Paper I we suggest an approximate gas density regime for the extraplanar dust features is $\sim 1-10 \mathrm{~cm}^{-3}$, assuming Galactic gas-to-dust relationships. At $z \approx 1 \mathrm{kpc}$ in NGC 891 the expected average density of electrons associated with the DIG is $\left\langle n_{e}\right\rangle \sim 0.06$ $\mathrm{cm}^{-3}$ (Rand 1997), with the true local density being perhaps $0.2-0.3$ depending on the filling factor. Given our lower limits to the implied column densities of the features listed in Table these structures would have to extend $>500-1000 \mathrm{pc}$ along the line of sight to have densities as low as the DIG. In most cases this is a significantly larger extent than the observed dimensions in the plane of the sky. It seems unlikely that the structures we observe extend that far along the line of sight. Work to be presented in a future publication (Howk \& Savage 1999a) suggests that not all of the absorbing dust features have associated $\mathrm{H} \alpha$ emission. If these high- $z$ dust clouds have gas densities as high as $1 \mathrm{~cm}^{-3}$ and are ionized, they would have very large $\mathrm{H} \alpha$ emission measures compared with the surrouding DIG. Thus the dust we see in absorption

may be a denser, neutral (and possibly molecular) "phase" of the ISM at high- $z$ than is traced through the $\mathrm{H} \alpha$ emission.

The dust-bearing clouds seen at high- $z$ may represent a stable dense phase within a multi-phase ISM at large distances from the plane (e.g., Wolfire et al. 1995b; McKee \& Ostriker 1977). Consideration of the thermal equilibrium of a medium can under the right conditions yield multiple stable equilibria. Thus regions of vastly different densities and temperatures can coexist in pressure equilibrium with one another. The range in parameter space (pressure and density) over which a multiphase medium is stable depends 
on the detailed heating and cooling processes of the medium (cf., Wolfire et al. 1995a). In particular the existence of a stable multiphase medium requires the pressure of the medium be in an appropriate range. At higher pressures only the dense phase is the stable, while at low pressures the low-density phase dominates.

For spiral galaxies having dusty thick disk gas with a requisite pressure, the formation of highlystructured clouds, such as those observed in our WIYN images, may be caused by a relaxation to the stable thermal equilibrium state of the medium. The presence of dust grains is extremely important for determining the conditions of the medium given the heating provided by photoelectron emission from dust and the cooling through thermal FIR radiation. Dust incorporates elements such as C, O, Si and Fe that might otherwise provide significant gas-phase coolants through IR fine structure emission lines.

Wolfire et al. (1995a) discuss the minimum required pressures to provide a thermally stable dense phase of the ISM in various environments. For a medium with standard Milky Way disk abundances, radiation fields (far-UV and X-ray), and dust to gas ratios, Wolfire et al. find a minimum pressure $P^{\text {min }} / k \approx 1000$ $\mathrm{K} \mathrm{cm}{ }^{-3}$ is required for the existence of a dense phase, assuming a cloud with $N_{\mathrm{H}} \approx 10^{19} \mathrm{~cm}^{-2}$. The column density of the cloud is important because the heating and ionization rates due to soft X-rays and far-UV radiation are sensitive to the absorbing column density. For clouds of order $N_{\mathrm{H}} \approx 10^{20} \mathrm{~cm}^{-2}$, closer to those observed in this work, they find $P^{\min } / k \approx 600 \mathrm{~K} \mathrm{~cm}^{-3}$. Other factors are important for such considerations, including the strength of the interstellar radiation field and the gas to dust ratio; in the case of the high- $z$ material discussed here these factors all tend to decrease the minumum pressure required for a stable dense gas phase, with the exception of possible variations in the $\mathrm{C}$ and $\mathrm{O}$ abundances (see Wolfire et al. 1995a).

Are the pressures at high- $z$ in our target galaxies sufficiently high to support a stable dense phase of the ISM? In the case of NGC 891, the best studied of these galaxies, the answer is yes. Observations of X-ray and $\mathrm{H} \alpha$ emission from NGC 891 have provided some information on the electron density distribution at high- $z$ (Bregman \& Houck 1997 and Rand 1997, respectively). Bregman \& Houck (1997) characterize the X-ray emitting gas with a temperature $T \sim 3.5 \times 10^{6} \mathrm{~K}$, central density $n_{o} \sim 0.026 \mathrm{~cm}^{-3}$, and a Gaussian scale height perpendicular to the plane of $h_{z} \sim 3.5 \mathrm{kpc}$. The radial scale-length of this gas is thought to be $h_{R} \sim 4.1 \mathrm{kpc}$. Thus the midplane pressure should be of order $P / k \sim 10^{5}$ and half that value at $R \approx 2 \mathrm{kpc}$, $z \approx 1.0 \mathrm{kpc}$. Even at $R \sim 8 \mathrm{kpc}$, comparable to the sun's distance from the Galactic center, the expected pressure of the hot ISM according to the Bregman \& Houck fits should be $P / k \sim 10^{4} \mathrm{~K} \mathrm{~cm}^{-3}$. Thus if the observed dusty clouds are immersed in the hot gaseous halo of this galaxy at $z \sim 1 \mathrm{kpc}$, the pressures are much greater than those required by the Wolfire et al. models. We find similar results if the clouds are embedded in the high- $z$ DIG. At $z \sim 1 \mathrm{kpc}$ the average density of electrons in the thick ionized gas layer of NGC 891 should be of order $\left\langle n_{e}\right\rangle \sim 0.06 \mathrm{~cm}^{-3}$, according to the distribution derived by Rand (1997). If we assume nebular temperatures for this gas, in this case $T_{e} \sim 8000 \mathrm{~K}$, and an ionization fraction $x_{e} \equiv n_{e} / n_{\mathrm{H}} \sim 0.8$ (Rand 1998), then we find $P / k \sim 1100 \mathrm{~K} \mathrm{~cm}^{-3}$ at $z \sim 1 \mathrm{kpc}$. Again this is sufficient to make the presence of dense gas in pressure equilibrium at high- $z$ reasonable, though not as comfortably as for the hot gas. If we assume a volume filling factor of $f \sim 0.2$, making the local electron density of the gas $n_{e} \sim 0.3 \mathrm{~cm}^{-3}$, the appropriate pressure is more like $P / k \sim 5500 \mathrm{~K} \mathrm{~cm}^{-3}$. Though we have not discussed the effects of differing abundances, gas to dust ratios, and radiation fields (which are discussed more fully for high- $z$ gas in Wolfire et al. 1995b), the expected pressures at heights $z \sim 1 \mathrm{kpc}$ in NGC 891 seem sufficiently high for the presence of a stable dense medium. Note that non-thermal pressures may also be important.

Assuming the observed dust structures represent a dense phase of the ISM at high- $z$, one can estimate the thermal crossing times for these structures. This allows us to roughly estimate the expected lifetime 
of a cloud assuming no sources of confinement. Rough estimates of the densities for our cloud structures, using our column density estimates and minor axis size scales, suggest densities of order $n_{\mathrm{H}} \sim 1-10 \mathrm{~cm}^{-3}$. At pressures characteristic of the DIG at high- $z$, e.g., $P / k \sim 1100-5500 \mathrm{~K} \mathrm{~cm}^{-3}$, this corresponds to temperatures of order $T \sim 100-5500 \mathrm{~K}$ (assuming pressure equilibrium), with lower temperatures being more likely if these structures truly represent a dense phase of the ISM. If we assume minor axis sizes of order $100 \mathrm{pc}$, the thermal crossing times for this range of temperatures is of order $10^{7}-10^{8}$ years. This is similar to the expected circulation timescales for a parcel of gas travelling from the disk to halo and back (e.g., Houck \& Bregman 1990; Norman \& Ikeuchi 1989). The timescales may be longer than this if magnetic fields are present. This suggests that these structures are not simply transient, but could indeed be long lived.

If the cool dust clouds are formed from thermal instabilities in the hot or warm ionized medium at high- $z$ it implies that dust must survive in the DIG and/or the hot gas. This is not an unreasonable scenario. Howk \& Savage (1999d) have recently shown that dust must exist in the warm ionized medium of the Milky Way based upon the gas-phase abundances of Al and S. And while there is little observational information on the dust content of the hot ISM, theoretical calculations suggest that grains are able to survive the thermal sputtering in such a harsh environment for as much as $10^{9}$ years (Ferrara et al. 1991).

A scenario in which the observed high- $z$ dusty clouds represent the dense phase of a multi-phase ISM at high- $z$, like the models discussed in Paper I, requires a large star formation rate in the underlying disk to provide both the material at high- $z$ and the requisite pressure. Thus fountain- or chimney-like outflows may cause a region of relatively high pressure just above the thin disk that can enable the formation of dense cloud condensations in the range of heights where we observe the absorbing structures considered in this work. If thermal equilibrium considerations drive the in situ formation of the observed dusty clouds at high- $z$, a natural consequence of the large required pressure may be a large emissivity in the $\mathrm{H} \alpha$ line from the DIG. The intensity of $\mathrm{H} \alpha$ emission from a parcel of gas is proportional to the product $n_{p} n_{e}$, or proportional to $n_{e}^{2}$ in a pure hydrogen gas, where $n_{p}$ and $n_{e}$ are the proton and electron densities, respectively. If we assume the DIG exists within a range of temperatures $T \sim 6000-10000 \mathrm{~K}$, the relatively large pressure required to confine the dense clouds is manifested in a large density, and hence fuels a large $\mathrm{H} \alpha$ emissivity. Thus galaxies that have high- $z$ material with thick disk pressures large enough to support a dense phase of the ISM may naturally be expected to show detectable $\mathrm{H} \alpha$ emission at high- $z$.

\subsubsection{High-z Star Formation}

It is interesting that many of the dust features we see in our target galaxies are of similar sizes and estimated masses to giant molecular clouds in the disk of the Milky Way. As we suggested earlier, the observed dust features may indeed contain molecular material, depending on the true gas to dust ratio and interstellar radiation field at high- $z$. Given this similarity it is possible stars may be presently forming from the high- $z$ material observed in many of our galaxies. Indeed, we have found evidence for high- $z$ $(0.6 \lesssim z \lesssim 1.0 \mathrm{kpc})$ H II regions in NGC 891 and NGC 4013 (Howk \& Savage 1999c). These regions of ionized gas are presumably powered by early-type stars, though spectroscopy is needed to confirm the classification of these objects as H II regions. Such high- $z$ H II regions have been noted by other authors for the galaxies NGC 4244 (Walterbos 1991) and NGC 55 (Ferguson, Wyse, \& Gallagher 1996). It would be worthwhile to carefully search for molecular emission (e.g., $\mathrm{CO}$ ) at high- $z$ in the galaxies that do show these dust structures. 


\subsection{Milky Way Analogs}

Are there structures in the Milky Way that are comparable to those observed here? This question is complicated by the difficulties of identifying structures given our position in the disk of the Milky Way. Koo, Heiles, \& Reach (1992) have identified Galactic structures they term "worms" seen in both H I and IRAS maps of the galaxy. These dusty filaments may be similar to the dusty clouds we see, though it is unclear if their vertical extents are as great as the structures seen in our sample of galaxies. The Draco cloud, an intermediate-velocity $\mathrm{H}$ I and molecular cloud of great interest since it absorbs background X-rays (Burrows \& Mendenhall 1991), has recently been shown to lie at a height $300 \lesssim z \lesssim 400$ pc by Gladders et al. (1998). This cloud may be similar in column density to the structures we discuss, but its mass and height above the plane are significantly less than the structures we identify. Callaway et al. (1999) report on H I observations of a Galactic supershell in the inner galaxy (the "Scutum Supershell"). This structure stretches to heights $z \lesssim 600$ pc from the midplane and contains some $\sim 10^{5} \mathrm{M}_{\odot}$, with an estimated potential energy of $\Omega \gtrsim 10^{52}$ ergs. Furthermore, a high- $z$ cloud $(z \sim 600 \mathrm{pc})$ containing $\sim 4 \times 10^{4} \mathrm{M}_{\odot}$ of material seems to be associated with this structure. The H I column density of this high- $z$ cloud is of order $3 \times 10^{20} \mathrm{~cm}^{-2}$, and its diameter is $\sim 170$ pc. A structure such as the Scutum Supershell, or perhaps the high- $z$ cloud associated with it, would be visible in our galaxies, provided there was little stellar light along the line of sight arising in front it. Another possible Galactic analog may be the Low-Latitude Intermediate Velocity Arch (LLIV Arch; Kuntz \& Danly 1996). Wakker et al. (1996) have shown this structure to lie at a height $1.1 \lesssim z \lesssim 3.0 \mathrm{kpc}$ from the plane of our Galaxy. With peak column densities of a few times $10^{20}$ $\mathrm{cm}^{-2}$, and a total mass $\gtrsim 10^{5}-10^{6} \mathrm{M}_{\odot}$ (Kuntz \& Danly 1996; Wakker et al. 1996), this feature might be detectable if placed in our target galaxies. Given its relatively low column density (and its unknown dust content) it might only be detectable if it were in front of most of the starlight.

\subsection{High- $z$ Opacity Distribution}

One implication of the observed dust structures in many of our target galaxies is that dust opacity cannot be neglected at heights $z \lesssim 1.5 \mathrm{kpc}$ from the midplane. In Paper I we estimated values of $0.8 \lesssim A_{\mathrm{V}} \lesssim 1.8 \mathrm{mag}$. for a sample of absorbing structures at heights $0.4 \lesssim z \lesssim 1.5 \mathrm{kpc}$ in NGC 891 . We have found similar values for absorbing structures in NGC 4013 (e.g., Howk \& Savage 1999c). Intercepting only one of these features along the line of sight provides an optical depth $\tau_{\mathrm{V}} \sim 1$ (to light arising behind it) in the visual wavebands. The complicated and widespread distribution of absorbing structures in many of these galaxies suggests that an observer may expect to intercept more than one of these features along sightlines having heights up to $z \sim 1 \mathrm{kpc}$, and perhaps further. The true form of the $z$-distribution of dust opacity in edge-on galaxies may be poorly constrained by many of the current fits to the vertical light distribution. The presence of a dusty thick disk of material with relatively large scale height (e.g., $\sim 1 \mathrm{kpc}$ ) can create problems for fits to the one- or two-dimensional light profiles of spiral galaxies. The opacity distribution caused by thin+thick disk distributions of dust can be relatively well-fit by a single component dust model (K. Wood, private communication), but the resulting scale height estimate of the opacity is larger (smaller) than that of the true thin (thick) disk opacity. Thus previous estimates for the dust scale heights in galaxies that show evidence for significant high-z opacity may need to be questioned.

If the gas-to-dust ratio in these dust structures is similar to that found in the Galactic disk, the opacity to X-ray emission could also be quite large. For example the optical depths at $0.25,0.5$ and $1.0 \mathrm{keV}$ associated with neutral disk material having $\tau_{V}=1.0$ in our Galaxy are $\tau(0.25,0.5,1.0 \mathrm{keV}) \approx(8.0,1.2$, and 0.5$)$, 
respectively (B. Benjamin, private communication). However, the distribution of opacity along a given sightline through a dusty thick disk of material is likely quite patchy, making the transfer of radiation more difficult to account for when attempting a correction for absorption. Studies of the distribution of material traced by X-ray, ultraviolet, or optical light should take care to examine how the presence of dust having optical depth unity at visual wavelengths to heights $z \sim 1 \mathrm{kpc}$ affect their results.

\section{SUMMARY}

We present the results of a high resolution ( 0.6 to $\sim 1^{\prime \prime} 0$ ) imaging survey, using the WIYN 3.5-m telescope, of nearby edge-on galaxies, the purpose of which is to search for high- $z$ dust in absorption against the background stellar light of our target galaxies. This work follows from the identification of hundreds of high- $z$ absorbing structures seen in the galaxy NGC 891 by Howk \& Savage (1997). A summary of our major results is as follows.

1. Inhomogeneously distributed (clumped) high- $z$ dust is a common property of $L_{*}$ spiral galaxies; five of seven such galaxies in our final sample exhibit high- $z(z \gtrsim 400 \mathrm{pc})$ dust structures.

2. There is a close correlation between the presence of high- $z$ DIG and dust in our sample. Of those galaxies that have been searched for high- $z \mathrm{H} \alpha$ emission (nine total, seven from our final sample), all that show such emission also show observable dust structures at high- $z$ (seven of nine, or five of seven drawn from our final sample), none of those galaxies lacking $\mathrm{H} \alpha$ emission at high- $z$ show evidence for dusty material far above the disk (two of nine, or two of seven from our final sample). This correlation may be due to a common driver of high- $z$ material and is probably associated with vigorous star formation in the underlying disk.

3. We derive the properties of several individual dust structures, which show sizes of order $50-100 \times 100-400 \mathrm{pc}$. In general we find apparent extinctions $a_{V} \sim 0.2-0.9$, though the true V-band extinction may be significantly greater than these values. Assuming Galactic gas to dust ratios are appropriate, the masses of these structures exceed $\sim 10^{5} \mathrm{M}_{\odot}$. The potential energies of these structures are likely quite large, of order $\Omega \gtrsim 10^{52}$ ergs relative to $z=0$. The dust features exhibit a wide range of morphologies. One of the more impressive examples is seen above the bulge of NGC 4217 (NGC 4217:D -016 + 015); this structure appears as a loop of diameter $\sim 0.8 \mathrm{kpc}$, maximum height $z \sim 1.3 \mathrm{kpc}$, and total mass $>5 \times 10^{5} \mathrm{M}_{\odot}$.

4. We comment on the possible mechanisms responsible for creating and shaping the observed dust structures. Possible mechanisms include hydrodynamical flows (e.g., galactic fountains or bores and chimney models), flows assisted or driven by magnetic fields or radiation pressure, and in situ formation of high- $z$ clouds from cooling high- $z$ gas. Three of our galaxies have close companions; in these cases the high- $z$ gas and dust may be caused by galaxy interactions, either through triggered star formation or tidal stripping.

5. We discuss in detail the possibility that the dusty clouds we observe represent a dense phase of the high- $z$ ISM in pressure equilibrium with the extraplanar warm ionized or hot medium. Rough calculations for the best-studied galaxy NGC 891 suggest that the high- $z$ ISM in this galaxy satisfies the requisite conditions for support of a dense phase (e.g., Wolfire et al. 1995a,b). Our rough estimates for the density of the observed structures are compatable with the Wolfire et al. results for high 
column density clouds. We discuss the possibility that star formation may be occuring in these dense high- $z$ clouds.

It is a pleasure to thank the operators at WIYN who, through their expertise, have made this survey possible: C. Corson, W. Hughes, C. Mackey, and G. Rosenstein. We also thank T. Tripp for sharing some of his WIYN observing time. JCH thanks M. de Avillez and R. Dettmar for enlightening discussions that have helped this work. This research has made use of the NASA/IPAC Extragalactic Database (NED) which is operated by the Jet Propulsion Laboratory, California Institute of Technology, under contract with the National Aeronautics and Space Administration. Our work has also made use of the SIMBAD

database, operated at CDS, Strasbourg, France. JCH recognizes support from a NASA Graduate Student Researcher Fellowship under grant number NGT-5-50121.

\section{REFERENCES}

Alton, P.B., Bianchi, S., Rand, R.J., Xilouris, E.M., Davies, J.I., \& Trewhella, M. 1998, ApJ, 507, L125

de Avillez, M.A., Berry, D.L., \& Kahn, F.D. 1998, in Lecture Notes in Physics, v. 506: The Local Bubble and Beyond, eds. D. Breitschwerdt, M.J. Freyberg, \& J. Truemper (Berlin: Springer-Verlag), p. 495

Bohlin, R.C., Savage, B.D. \& Drake, J.F. 1978, ApJ, 224, 132

Bottema, R. 1995, A\&A, 295, 605

Bottema, R. 1996, A\&A, 306, 345

Bregman, J.N. 1980, ApJ, 236, 577

Bregman, J.N., \& Houck, J.C. 1997, ApJ, 485, 159

Burrows, D.N., \& Mendenhall, J.A. 1991, Nature, 351, 629

Callaway, M. et al. 1999, in preparation.

Cardelli, J.A., Clayton, G.C. \& Mathis, J.S. 1989, ApJ, 345, 245

Cox, P., \& Mezger, P.G. 1989, Astron. Astrophys. Rev., 1, 49

Dahlem, M., Heckman, T.M., Fabbiano, G., Lehnert, M.D., \& Gilmore, D. 1996, ApJ, 461, 724

Davies, J.I., Alton, P., Bianchi, S., \& Trewhella, M. 1998, MNRAS, 300, 1006

Dettmar, R.-J. 1990, A\&A, 232, L15

Dettmar, R.-J. 1998, in Lecture Notes in Physics, v. 506: The Local Bubble and Beyond, eds. D. Breitschwerdt, M.J. Freyberg, \& J. Truemper (Berlin: Springer-Verlag), p. 527

Dettmar, R.-J., \& Schulz, H. 1992, A\&A, 254, L25

de Vaucouleurs, G., de Vaucouleurs, A., Corwin, H.G., Buta, R.J., Paturel, G., \& Fouque, P. 1991, Third Reference Catalogue of Bright Galaxies, (New York: Springer-Verlag)

Dumke, M., Krause, M., Wielebindki, R. \& Klein, U. 1995, A\&A, 302, 691

Fabbiano, G., Heckman, T.M., \& Keel, W.C. 1990, ApJ, 355, 442

Ferguson, A.M.N., Wyse, R.F.G., \& Gallagher, J.S. 1996, AJ, 112, 2567

Ferrara, A. 1993, ApJ, 407, 157 
Ferrara, A., Ferrini, F., Barsella, B. \& Franco, J. 1991, ApJ, 381, 137

Franco, J., Ferrini, F., Barsella, B., \& Ferrara, A. 1991, ApJ, 366, 443

Fullmer, L., \& Lonsdale, C. 1989, Cataloged Galaxies and Quasars Observed in the IRAS Survey, Version 2 (Pasadena: Jet Propulsion Laboratory)

García-Burillo, S., Combes, F., \& Neri, R. 1999, A\&A, in press.

Gladders, M.D. et al. 1998, ApJ, 507, L161

Golla, G., Dettmar, R.-J., \& Domgörgen, H. 1996, A\&A, 313, 439

Heiles, C. 1979, ApJ, 229, 533

Houck, J.C. \& Bregman, J.N. 1990, ApJ, 352, 506

Howk, J.C., \& Savage, B.D. 1997, AJ, 114, 2463 (Paper I)

Howk, J.C., \& Savage, B.D. 1999a, in preparation.

Howk, J.C., \& Savage, B.D. 1999b, in preparation.

Howk, J.C., \& Savage, B.D. 1999c, in The Physics and Chemistry of the Interstellar Medium: Proceedings of the 3rd Colögne-Zermatt Symposium, ed. V. Ossenkopf, in press.

Howk, J.C., \& Savage, B.D. 1999d, ApJ, in press.

Hummel, E., Beck, R. \& Dahlem, M. 1991, A\&A, 248, 23

Keppel, J.W., Dettmar, R.-J., Gallagher, J.S. \& Roberts, M.S. 1991, ApJ, 374, 507

Koo, B.-C., Heiles, C. \& Reach, W.T. 1992, ApJ, 390, 108

Kuntz, K.D., \& Danly, L. 1996, ApJ, 457, 703

McKee, C.F. \& Ostriker, J.P. 1977, ApJ, 218, 148

Morrison, H., Boroson, T., \& Harding, P. 1994, AJ, 108, 1191

Naeslund, M., \& Joersaeter, S. 1997, A\&A, 325, 915

Norman, C.A. \& Ikeuchi, S. 1989, ApJ, 345, 372

Parker, E.N. 1966, ApJ, 145, 811

Parker, E.N. 1992, ApJ, 401, 137

Pildis, R.A., Bregman, J.N. \& Schombert, J.M. 1994, ApJ, 427, 160

Rand, R.J. 1996, ApJ, 462, 712

Rand, R.J. 1997, ApJ, 474, 129

Rand, R.J. 1998, in The Interstellar Medium in Galaxies, ed. J.M. van der Hulst (Dordrecht: Kluwer), p. 105

Rand, R.J., Kulkarni, S.R. \& Hester, J.J. 1990, ApJ, 352, L1

Rand, R.J., Kulkarni, S.R. \& Hester, J.J. 1992, ApJ, 396, 97

Rhee, M.-H., \& van Albada, T.S. 1996, A\&AS, 115, 507

Roberts, M.S., \& Haynes, M.P. 1994, ARA\&A, 32, 115

Rupen, M. 1991, AJ, 102, 48

Sánchez-Saavedra, M.L., Battaner, E., \& Florido, E. 1990, MNRAS, 246, 458 
Sancisi, R. 1976, A\&A, 53, 159

Sancisi, R. \& Allen, R.J. 1979, A\&A, 74, 73

Scoville, N.Z., \& Sanders, D.B. 1987, in Interstellar Processes, edited by D.J. Hollenbach \& H.A. Thronson, Jr. (Reidel, Dordrecht), p. 21

Scoville, N.Z., Thakkar, D., Carlstrom, J.E. \& Sargent, A.I. 1993, ApJ, 404, L59

Shang, Z., et al. 1998, ApJ, 504, L23

Shapiro, P. \& Field, G.B. 1976, ApJ, 205, 762

Sukumar, S. \& Allen, R.J. 1991, ApJ, 382, 100

Swaters, R.A., Sancisi, R. \& van der Hulst, J.M. 1997, ApJ, 491, 140

Teerikorpi, P., Bottinelli, L., Gouguenheim, L., \& Paturel, G. 1992, A\&A, 260, 17

Tully, R.B. 1988, Nearby Galaxies Catalog, (Cambridge: Cambridge University Press)

Vogler, A., Pietsch, W., \& Kahabka, P. 1996, A\&A, 305, 74

Wakker, B., Howk, C., Schwarz, U., van Woerden, H., Beers, T., Wilhelm, R., Kalberla, P. \& Danly, L. 1996, ApJ, 473, 834

Walterbos, R.A.M. 1991, in The Interstellar Disk-Halo Connection in Galaxies, ed. H. Bloemen (Dordrecht: Kluwer), p. 223

Wilding, T., Alexander, P., \& Green, D.A. 1993, MNRAS, 263, 1075

Weliachew, L., Sancisi, R., \& Guélin, M. 1978, A\&A, 65, 37

Wolfire, M.G., Hollenbach, D., McKee, C.F., Tielens, A.G.G.M., \& Bakes, E.L.O. 1995a, ApJ, 443, 152

Wolfire, M.G., McKee, C.F., Hollenbach, D., \& Tielens, A.G.G.M. 1995b, ApJ, 453, 673 
Table 1. Edge-On Galaxy Targets ${ }^{\mathrm{a}}$

\begin{tabular}{lcccccccc}
\hline \hline \multicolumn{1}{c}{ Name } & $\begin{array}{c}\mathrm{RA} \\
{[\mathrm{J} 2000]}\end{array}$ & $\begin{array}{c}\text { Dec. } \\
{[\mathrm{J} 2000]}\end{array}$ & $\begin{array}{c}D_{25} \\
{[\operatorname{arcmin}]}\end{array}$ & $\begin{array}{c}\mathrm{V}_{\text {rad }} \\
{\left[\mathrm{km} \mathrm{s}^{-1}\right]}\end{array}$ & $\begin{array}{c}W_{20} \\
{\left[\mathrm{~km} \mathrm{~s}^{-1}\right]}\end{array}$ & $\begin{array}{c}\text { Dist. } \\
{[\mathrm{Mpc}]}\end{array}$ & $\begin{array}{c}L_{F I R} \\
{\left[10^{9} \mathrm{~L}_{\odot}\right]}\end{array}$ & Type \\
\hline NGC 891 & 022233 & +422048 & 12.2 & 528 & 471 & 9.6 & 9.9 & $\mathrm{Sb}$ \\
NGC 3628 & 112016 & +133522 & 14.8 & 843 & 476 & 7.7 & 5.2 & $\mathrm{Sb}$ \\
NGC 4013 & 115832 & +435648 & 4.7 & 835 & 407 & 17 & 3.7 & $\mathrm{Sb}$ \\
NGC 4157 & 121105 & +502910 & 7.0 & 774 & 413 & 17 & 8.5 & $\mathrm{Sbc}$ \\
NGC 4183 & 121318 & +434155 & 5.1 & 930 & 249 & 17 & $<0.2$ & $\mathrm{Scd}$ \\
NGC 4217 & 121551 & +470532 & 5.1 & 1026 & 431 & 17 & $<0.2$ & $\mathrm{Sb}$ \\
NGC 4302 & 122142 & +143605 & 4.7 & 1149 & 377 & 17 & $\lesssim 3.3$ & $\mathrm{Sc}$ \\
NGC 4517 & 123228 & +002323 & 9.5 & 1131 & 164 & 9.8 & 1.0 & $\mathrm{Sc}$ \\
NGC 4565 & 123621 & +255905 & 16.2 & 1220 & 525 & 9.7 & 1.6 & $\mathrm{Sb}$ \\
NGC 4631 & 124208 & +323228 & 14.7 & 632 & 315 & 6.9 & 4.1 & $\mathrm{Sc}$ \\
NGC 4634 & 124240 & +141747 & 2.6 & 297 & 280 & $19^{\mathrm{b}}$ & 3.2 & $\mathrm{Sc}$ \\
NGC 5907 & 151554 & +561946 & 11.2 & 667 & 485 & 15 & 5.0 & $\mathrm{Sc}$ \\
\hline
\end{tabular}

${ }^{a}$ The properties presented here were taken from the NED database with the exception of the distances, which were taken from Tully (1988) and the far-infrared luminosities, $L_{F I R}$, which were derived using the adopted distances and the far-infrared fluxes of Fullmer \& Lonsdale (1989). The width of the H I profile at $20 \%$ of the peak flux, $W_{20}$, is taken from the RC3 (de Vaucouleurs et al. 1991).

b The distance to NGC 4634 is taken from Teerikorpi et al. (1992) and is based on a Tully-Fisher magnitude-line width relationship. 
Table 2. Log of Observations ${ }^{\mathrm{a}}$

\begin{tabular}{ccccc}
\hline \hline Galaxy & Filter & Date & $\begin{array}{c}\text { Seeing } \\
\text { [arcsec] }\end{array}$ & $\begin{array}{c}\text { Resolution } \\
{[\mathrm{pc}]}\end{array}$ \\
\hline NGC 891 & B & 1997 Aug. 29 & 0.6 & 27 \\
& V & 1997 Aug. 30 & 0.6 & 27 \\
NGC 3628 & B & 1998 Mar. 02 & 0.9 & 34 \\
& V & 1998 Mar. 02 & 0.9 & 34 \\
NGC 4013 & B & 1997 Apr. 08 & 0.6 & 49 \\
& V & 1997 Apr. 08 & 0.5 & 41 \\
NGC 4157 & B & 1998 Apr. 17 & 0.9 & 74 \\
& V & 1998 Apr. 16 & 1.0 & 82 \\
NGC 4183 & B & 1998 Apr. 17 & 1.0 & 82 \\
& V & 1998 Apr. 16 & 1.0 & 82 \\
NGC 4217 & B & 1998 Apr. 17 & 0.8 & 66 \\
& V & 1998 Apr. 17 & 0.8 & 66 \\
NGC 4302 & B & 1997 May. 12 & 0.9 & 74 \\
& V & 1998 Apr. 17 & 0.8 & 66 \\
NGC 4517 & B & 1998 Apr. 17 & 0.9 & 41 \\
& V & 1998 Apr. 17 & 1.1 & 50 \\
NGC 4565 & B & 1997 Apr. 07 & 0.8 & 38 \\
& V & 1996 Dec. 03 & 0.8 & 38 \\
& V & 1997 Apr. 07 & 1.0 & 53 \\
NGC 4631 & B & 1998 Apr. 17 & 0.8 & 27 \\
& V & 1998 Apr. 16 & 1.0 & 33 \\
NGC 4634 & B & 1998 Apr. 17 & 1.0 & $19:$ \\
& V & 1998 Apr. 17 & 0.8 & $16:$ \\
& B & 1997 Apr. 06 & 1.3 & 95 \\
& V & 1998 Apr. 17 & 1.5 & 110 \\
\hline
\end{tabular}

${ }^{a}$ All observations have 900s exposure times except where noted.

${ }^{\mathrm{b}}$ Linear resolution of the observations assuming the distance given in Table 1.

${ }^{\mathrm{c}}$ The exposure time for the NGC $4013 \mathrm{~V}$-band data presented here is $600 \mathrm{~s}$.

${ }^{\mathrm{d}}$ The NGC $4565 \mathrm{~V}$-band data were split between two nights. The 1996 Dec. data have a 300s exposure time; the 1997 Apr. data have 600s exposure time. We have coadded the data after smoothing the Dec. data to 1."0 resolution. 
Table 3. Dust and DIG Properties for Edge-On Galaxy Sample

\begin{tabular}{|c|c|c|c|c|c|c|}
\hline Name & $\begin{array}{c}\text { Final } \\
\text { Sample }^{\mathrm{a}}\end{array}$ & $\begin{array}{c}\mathrm{L}_{F I R} / \mathrm{D}_{25}^{2} \\
\left(10^{40} \mathrm{erg} / \mathrm{s} \mathrm{kpc}^{2}\right)\end{array}$ & $\begin{array}{l}\text { High-z } \\
\text { Dust }^{b}\end{array}$ & $\begin{array}{l}\text { High-z } \\
\text { DIG }^{c}\end{array}$ & $\begin{array}{l}\text { DIG } \\
\text { Ref. }\end{array}$ & $\begin{array}{c}\text { DIG } \\
\text { Morphology }^{\mathrm{d}}\end{array}$ \\
\hline NGC 4634 & & 5.9 & $\bullet$ & $\bullet$ & 1 & Very bright; extended \\
\hline NGC 891 & $\sqrt{ }$ & 3.3 & $\bullet$ & $\bullet$ & 2,3 & Bright; diffuse+filaments \\
\hline NGC 4157 & & 2.7 & - & & & \\
\hline NGC 4013 & $\sqrt{ }$ & 2.6 & $\bullet$ & $\bullet$ & 4 & Faint; diffuse \\
\hline NGC 4302 & $\sqrt{ }$ & $\lesssim 2.3$ & $\bullet$ & $\bullet$ & 4 & Faint; diffuse \\
\hline NGC 3628 & $\sqrt{ }$ & 1.8 & $\bullet$ & $\bullet$ & 5 & \\
\hline NGC 4631 & & 1.8 & $\bullet$ & $\bullet$ & 6 & Bright; diffuse \\
\hline NGC 5907 & $\sqrt{ }$ & 0.8 & O & O & 4 & \\
\hline NGC 4517 & & 0.5 & O & & & \\
\hline NGC 4565 & $\sqrt{ }$ & 0.3 & O & O & 6 & \\
\hline NGC 4217 & $\sqrt{ }$ & $<0.12$ & $\bullet$ & $\bullet$ & 4 & 2 faint patches \\
\hline NGC 4183 & & $<0.12$ & - & & & \\
\hline
\end{tabular}

aSome of the galaxies we have imaged were excluded from our final sample due to low inclinations or low rotational velocities (see text). We have marked galaxies included in our final sample with a $\sqrt{ }$ mark.

${ }^{\mathrm{b}}$ denotes galaxies that exhibit high- $z$ dust in WIYN images; $\bigcirc$ denotes galaxies that do not exhibit high- $z$ dust in WIYN images. A few cases were ambiguous due to inclination effects; these are marked with - symbol.

c denotes galaxies with observable high- $z$ DIG; $\bigcirc$ denotes galaxies for which $\mathrm{H} \alpha$ searches have not shown detectable high- $z$ DIG.

${ }^{\mathrm{d}}$ After Dettmar (1998) and Rand (1996).

References. - (1) Dettmar (1998); (2) Dettmar (1990); (3) Rand, Kulkarni, \& Hester (1990); (4) Rand (1996); (6) Fabbiano, Heckman, \& Keel (1990); (5) Rand, Kulkarni, \& Hester (1992) 
Table 4. Properties of Individual High- $z$ Dust Features

\begin{tabular}{|c|c|c|c|c|c|c|c|c|c|}
\hline $\mathrm{ID}^{\mathrm{a}}$ & $\begin{array}{c}\text { R.A. } \\
{[\text { J2000] }}\end{array}$ & $\begin{array}{c}\text { Dec. } \\
{[\mathrm{J} 2000]}\end{array}$ & $\begin{array}{r}z^{\mathrm{b}} \\
{[\mathrm{pc}]}\end{array}$ & $\begin{array}{c}\text { Dimensions } \\
{[\mathrm{pc} \times \mathrm{pc}]}\end{array}$ & $\begin{array}{c}a_{B}{ }^{\mathrm{c}} \\
{[\mathrm{mag} .]}\end{array}$ & $\begin{array}{c}a_{V}{ }^{\mathrm{c}} \\
{[\mathrm{mag} .]}\end{array}$ & $\begin{array}{c}N_{\mathrm{H}}^{\mathrm{d}} \\
{\left[\mathrm{cm}^{-2}\right]}\end{array}$ & $\begin{array}{l}\operatorname{Mass}^{\mathrm{e}} \\
{\left[\mathrm{M}_{\odot}\right]}\end{array}$ & Morphology \\
\hline NGC 0891:D $-044+032^{\mathrm{f}}$ & 022229.1 & +422023 & 1450 & $50 \times 100$ & 0.85 & 0.60 & $>1 \times 10^{21}$ & $>1 \times 10^{5}$ & Elongated Cloud \\
\hline NGC 0891:D $-012-030^{\mathrm{f}}$ & 022235.2 & +422029 & 1350 & $60 \times 140$ & 0.44 & 0.32 & $>6 \times 10^{20}$ & $>1 \times 10^{5}$ & Cometary Cloud \\
\hline NGC 3628:D - $030-032$ & 112015.0 & +133442 & 1120 & $70 \times 160$ & 0.68 & 0.50 & $>1 \times 10^{21}$ & $>8 \times 10^{5}$ & Thin Sheet \\
\hline NGC 3628:D -053+ 039 & 112014.4 & +133558 & 1450 & $75 \times 210$ & 0.28 & 0.25 & $>5 \times 10^{20}$ & $>1 \times 10^{5}$ & Irr. Cloud \\
\hline NGC 4013:D +032- 012 & 115834.3 & +435652 & 1000 & $250 \times 300$ & 0.57 & 0.52 & $>1 \times 10^{21}$ & $>9 \times 10^{5}$ & Irr. Cloud \\
\hline NGC 4013:D - $001-032$ & 115831.4 & +435644 & 640 & $100 \times 230$ & 0.46 & 0.35 & $>6 \times 10^{20}$ & $>2 \times 10^{5}$ & Irr. Cloud \\
\hline NGC 4217:D $-016+015$ & 121548.6 & +470532 & 1260 & $130 \times 800^{\mathrm{g}}$ & 0.21 & 0.18 & $>3 \times 10^{20}$ & $>5 \times 10^{5}$ & Large Loop \\
\hline NGC 4302:D $-007+006$ & 122141.7 & +143544 & 500 & $150 \times 350$ & 0.36 & 0.31 & $>6 \times 10^{20}$ & $>4 \times 10^{5}$ & Irr. Cloud \\
\hline NGC 4302:D +029- 018 & 122143.5 & +143618 & 1450 & $130 \times 260$ & 0.60 & 0.45 & $>8 \times 10^{20}$ & $>3 \times 10^{5}$ & Irr. Cloud \\
\hline NGC 4302:D +038+ 009 & 122141.6 & +143627 & 710 & $130 \times 200$ & 0.68 & 0.54 & $>9 \times 10^{20}$ & $>5 \times 10^{5}$ & Irr. Cloud \\
\hline NGC 4565:D -064-016 & 123617.0 & +260000 & 700 & $210 \times 400$ & 1.20 & 0.90 & $>2 \times 10^{21}$ & $>1 \times 10^{6}$ & Vert. Column \\
\hline NGC 4634:D - $016-010$ & 124240.8 & +141733 & 1000 & $90 \times 180$ & 0.58 & 0.33 & $>6 \times 10^{20}$ & $>1 \times 10^{5}$ & Irr. Cloud \\
\hline
\end{tabular}

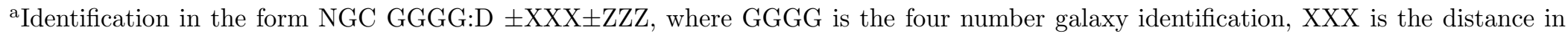
arcsec from the optical center of the galaxy traced along the major axis, and ZZZ is the distance in arcsec from the optical center of the galaxy traced along the minor axis (see text).

${ }^{\mathrm{b}}$ Projected height above the midplane, or the limit to which very extended features can be traced.

${ }^{\mathrm{c}}$ Apparent extinction for the BV wavebands in magnitudes, as defined in the text.

${ }^{\mathrm{d}}$ Approximate lower limit to the column density of material assuming Galactic extinction and gas-to-dust conversions and $R_{\mathrm{V}}=3.1$.

${ }^{\mathrm{e}}$ Approximate mass based upon the estimated column density and projected area. Includes a factor of 1.37 correction for He.

${ }^{\mathrm{f}}$ These two features in NGC 891 were discussed in Paper I as features 2 and 7 (NGC 0891:D $-044+032$ and NGC 0891:D -012 - 030, respectively). The potential energies of these structures relative to the midplane of NGC 891 were estimated to be $\Omega \sim 3 \times 10^{52}$ ergs in Paper I.

${ }^{\mathrm{g}}$ The dimensions given here represent the diameter of the loop at maximum extent, and the thickness of the walls of the loop. 
Fig. 1.- WIYN V-band images of NGC 891. The bottom panel shows the V-band image of this galaxy, while the top panel shows an unsharp masked version of the V-band data. The box at the lower left corner is $10^{\prime \prime} \times 10^{\prime \prime}$. At the distance of NGC $891,10^{\prime \prime}$ corresponds to $\sim 450 \mathrm{pc}$. North is to the top of the page and east to the left.

Fig. 2.- As Figure 1, but for the galaxy NGC 3628. At the distance of NGC 3628, 10" corresponds to $\sim 375$ pc.

Fig. 3.- As Figure 1, but for the galaxy NGC 4013. At the distance of NGC 4013, 10" corresponds to $\sim 825 \mathrm{pc}$.

Fig. 4.- As Figure 1, but for the galaxy NGC 4157. At the distance of NGC 4157, 10" corresponds to $\sim 825$ pc. This galaxy is not included in our final sample.

Fig. 5.- As Figure 1, but for the galaxy NGC 4183. In this case the unsharp masked image is shown on the left and the V-band on the right. At the distance of NGC 4183, 10" corresponds to $\sim 825$ pc. This galaxy is not included in our final sample.

Fig. 6.- As Figure 1, but for the galaxy NGC 4217. At the distance of NGC 4217, 10" corresponds to $\sim 825$ pc.

Fig. 7.- As Figure 1, but for the galaxy NGC 4302. In this case the unsharp masked image is shown on the left and the V-band on the right. At the distance of NGC 4302, 10" corresponds to $\sim 825 \mathrm{pc}$.

Fig. 8.- As Figure 1, but for the galaxy NGC 4517. At the distance of NGC 4517, 10" corresponds to $\sim 450 \mathrm{pc}$. This galaxy is not included in our final sample.

Fig. 9.- As Figure 1, but for the galaxy NGC 4565. At the distance of NGC 4565, $10^{\prime \prime}$ corresponds to $\sim 450$ pc. The unsharp mask image of this galaxy shows a slight artifact surrounding the location of the bright bulge of this galaxy.

Fig. 10.- As Figure 1, but for the galaxy NGC 4631. At the distance of NGC 4631, 10" corresponds to $\sim 335 \mathrm{pc}$. This galaxy is not included in our final sample. Many of the dust structures in this galaxy seem to be larger than the adopted FWHM of the smoothing Gaussian. Therefore some of the features visible in the V-band image (bottom panel) become less distinct in the unsharp mask (top panel).

Fig. 11.- As Figure 1, but for the galaxy NGC 4634. At the distance of NGC 4634, 10" corresponds to $\sim 920$ pc. This galaxy is not included in our final sample. The unsharp mask of this galaxy shows faint artifacts running mostly parallel to the disk that are caused by the extended thick stellar disk discussed in $\$ 4.2$. 
Fig. 12.- As Figure 1, but for the galaxy NGC 5907. At the distance of NGC 5907, 10" corresponds to $\sim 730$ pc.

Fig. 13.- Close-up views of individual dust features. These close-ups are taken from the unsharp-masked V-band images. The ID for each feature is given in a slightly compressed notation to save space, and the properties of each feature are summarized in Table 4 . We have lightly outlined each cloud. In a few cases we outline possible extensions of the cloud that were not used for calculating the values of Table 4 with dashed lines.

Fig. 14.- As Figure 13 but showing different structures.

Fig. 15.- As Figure 13 but showing different structures. 
This figure "Howk.fig1.jpg" is available in "jpg" format from: http://arxiv.org/ps/astro-ph/9902061v1 
This figure "Howk.fig2.jpg" is available in "jpg" format from: http://arxiv.org/ps/astro-ph/9902061v1 
This figure "Howk.fig3.jpg" is available in "jpg" format from: http://arxiv.org/ps/astro-ph/9902061v1 
This figure "Howk.fig4.jpg" is available in "jpg" format from: http://arxiv.org/ps/astro-ph/9902061v1 
This figure "Howk.fig5.jpg" is available in "jpg" format from: http://arxiv.org/ps/astro-ph/9902061v1 
This figure "Howk.fig6.jpg" is available in "jpg" format from: http://arxiv.org/ps/astro-ph/9902061v1 
This figure "Howk.fig7.jpg" is available in "jpg" format from: http://arxiv.org/ps/astro-ph/9902061v1 
This figure "Howk.fig8.jpg" is available in "jpg" format from: http://arxiv.org/ps/astro-ph/9902061v1 
This figure "Howk.fig9.jpg" is available in "jpg" format from: http://arxiv.org/ps/astro-ph/9902061v1 
This figure "Howk.fig10.jpg" is available in "jpg" format from: http://arxiv.org/ps/astro-ph/9902061v1 
This figure "Howk.fig11.jpg" is available in "jpg" format from: http://arxiv.org/ps/astro-ph/9902061v1 
This figure "Howk.fig12.jpg" is available in "jpg" format from: http://arxiv.org/ps/astro-ph/9902061v1 
This figure "Howk.fig13.jpg" is available in "jpg" format from: http://arxiv.org/ps/astro-ph/9902061v1 
This figure "Howk.fig14.jpg" is available in "jpg" format from: http://arxiv.org/ps/astro-ph/9902061v1 
This figure "Howk.fig15.jpg" is available in "jpg" format from: http://arxiv.org/ps/astro-ph/9902061v1 